\title{
Interleukin-3 and c-Kit/stem cell factor are required for normal eosinophil responses in mice infected with Strongyloides venezuelensis
}

Koichi Kimura ${ }^{1,2,3}$, Chang Ho Song ${ }^{1,2,4}$, Akhil Rastogi ${ }^{5}$, Glenn Dranoff ${ }^{6,7}$, Stephen J Galli ${ }^{1,2,8}$ and Chris S Lantz ${ }^{1,2,5}$

${ }^{1}$ Department of Pathology, Beth Israel Deaconess Medical Center, Boston, MA, USA; ${ }^{2}$ Department of Pathology, Harvard Medical School, Boston, MA, USA; ${ }^{3}$ Department of Pediatrics and Child Health, Kurume University School of Medicine, Kurume City, Japan; ${ }^{4}$ Department of Anatomy, Chonbuk National University Medical School, Jeonju, Republic of Korea; ${ }^{5}$ Department of Biology, James Madison University, Harrisonburg, VA, USA; ${ }^{6}$ Department of Medicine, Dana-Farber Cancer Institute, Boston, MA, USA; ${ }^{7}$ Department of Medicine, Harvard Medical School, Boston, MA, USA and ${ }^{8}$ Department of Pathology, Stanford University School of Medicine, Stanford, CA, USA

To evaluate the potential roles of Interleukin-3 (IL-3) and c-Kit, the tyrosine kinase receptor for stem cell factor (SCF), in eosinophil responses in vivo, we examined eosinophil numbers in uninfected or nematode-infected wild-type mice, IL-3-I- mice, and IL-3-I- mice that also have a marked reduction in SCF/C-Kit signaling (ie, $\mathrm{Kit}^{W} / \mathrm{Kit}^{\mathrm{W}-\mathrm{v}}$, IL-3-I- mice). We found no significant differences in the numbers of eosinophils in the blood, bone marrow or various tissues of IL-3-I-vs IL-3 $+I+$ mice, either at baseline or after the induction of bone marrow, blood or tissue eosinophilia in response to infection with Strongyloides venezuelensis (S.v.) or Nippostrongylus brasiliensis (N.b.). However, in mice with markedly impaired SCF/C-Kit signaling, IL-3 contributed significantly to the increased numbers of eosinophils that were observed in multiple tissues during S.v. infection, but not during infection with $N$.b.

Laboratory Investigation (2006) 86, 987-996. doi:10.1038/labinvest.3700458; published online 7 August 2006

Keywords: basophils; host defense; mast cells; Nippostrongylus brasiliensis; parasite immunity

Interleukin-3 (IL-3) can promote the development of various hematopoietic cells in vitro, through effects on both multipotential and more mature, lineagecommitted progenitor cells. ${ }^{1}$ While many studies have shown that IL-3 can enhance the development, survival and functional activation of mouse or human eosinophils in vitro, ${ }^{2-6}$ the importance of IL-3 in eosinophil development in vivo has been difficult to assess. In part, this reflects the fact that three cytokines, IL-3, IL-5 and GM-CSF, whose genes are closely linked and which all can be expressed by activated $\mathrm{T}$ cells or mast cells, express overlapping functions. ${ }^{7-10}$ For example, all three cytokines can

Correspondence: Dr SJ Galli, MD, Department of Pathology, Stanford University School of Medicine, L235, 300 Pasteur Drive, Stanford, CA 94305-5324, USA.

E-mail: sgalli@stanford.edu

Received 17 May 2005; revised 29 June 2006; accepted 30 June 2006; published online 7 August 2006 promote eosinophil development in vitro. ${ }^{5,6}$ Moreover, all three of these cytokines bind to cell surface receptors which are heterodimers consisting of the same 'common' $\beta$ chain $\left(\beta_{\mathrm{c}}\right)$; $^{3}$ this $\beta_{\mathrm{c}}$ forms high affinity receptors for IL-3, IL-5 or GM-CSF when paired with the appropriate specific ligand-binding $\alpha$ chain. ${ }^{11,12}$ Mice, but not humans, also express an IL-3-specific $\beta$ chain $\left(\beta_{\mathrm{IL}-3}\right){ }^{13-15}$ The mouse $\beta_{\mathrm{IL}-3}$ is $91 \%$ identical to the mouse $\beta_{\mathrm{c}}$ at the amino-acid level, but it does not form functional high-affinity heterodimers with the mouse IL-5 or GM-CSFspecific $\alpha$ chains. ${ }^{11,12}$

Work with mice which lack either of the two $\beta$ receptor genes ${ }^{7-9}$ as well as studies in IL-5 -/ - or IL-5R $\alpha$ chain $-/-$ mice $^{16,17}$ indicate that IL-3 cannot overcome the marked defect in eosinophil production that is associated with a lack of signaling via the receptor for IL-5. Nevertheless, four considerations prompted us to reassess the potential role of IL-3 in mouse eosinophil responses in vivo. First, because 
$\beta_{\mathrm{c}}-/-$ mice and $\beta_{\mathrm{c}}-/-\times$ IL-3 $-/-$ mice lack IL-5 signaling, and therefore have markedly impaired eosinophil production, such animals do not represent an optimal setting in which to search for potentially important effects of IL-3, for example, enhancement of IL-5-dependent eosinophil development. ${ }^{4,18}$ Second, we recently reported that eosinophil recruitment after i.p. allergen challenge in ragweed allergen-immunized mice was greater in IL-3 - / - mice than in IL-3 + / + mice. ${ }^{19}$ That IL-3 might, under certain circumstances, suppress eosinophil development and/or migration is supported by studies showing that mice deficient in both IL-3 and GM-CSF, but not single knockouts, exhibit increased numbers of circulating eosinophils. ${ }^{20}$ Third, we reported that while IL-3 -/- mice were normal in many aspects of hematopoiesis in vitro or in vivo ${ }^{21}$ as would have been predicted based on the phenotype of $\beta_{\mathrm{c}}-/-\times \mathrm{IL}-3-/-$ mice,${ }^{10} \mathrm{IL}-3-/-$ mice showed marked impairment of two important features of their Th2-associated hematopoietic response to infection with the nematode Strongyloides venezuelensis (S.v.): bone marrow basophil production and tissue mast cell hyperplasia. ${ }^{22}$ However, the eosinophil responses of these mice were not assessed. Fourth, stem cell factor (SCF), the ligand for the tyrosine kinase receptor c-Kit, has also been reported to enhance eosinophil development in vitro, but only when used in combination with other cytokines such as IL-3. ${ }^{23,24}$ SCF also can stimulate VLA-4-mediated adhesion of human eosinophils to fibronectin and VCAM-1 in vitro. ${ }^{25}$ SCF is required for physiological mast cell development in mice, as mast cells are essentially absent under baseline conditions in the tissues of $\mathrm{Kit}^{W} / \mathrm{Kit}^{W-\mathrm{V}}$ mice that have markedly reducing c-Kit signaling. ${ }^{26,27}$ However, by using $\mathrm{Kit}^{W /} / \mathrm{Kit}^{W-\mathrm{V}}$, IL-3 -/mice, we showed that the modest numbers of mast cells that can appear in the intestines or spleen of $\mathrm{Kit}^{W} / \mathrm{Kit}^{\mathrm{W-V}}$ mice infected with S.V. are dependent on IL-3. ${ }^{22}$ This work indicated that $\mathrm{Kit}^{W} / \mathrm{Kit}^{W-\mathrm{V}}$, IL-3 -/mice can be useful for revealing biological responses that may depend on both IL-3 and c-Kit/SCF signaling for their full expression.

We therefore used IL-3 - /- and wild-type mice, as well as $\mathrm{Kit}^{W} / \mathrm{Kit}^{W-\mathrm{V}}$, IL-3 -/- mice and the

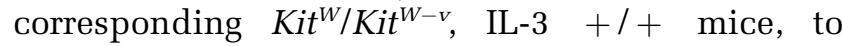
assess the extent to which IL-3 might act alone or in concert with c-Kit/SCF to promote eosinophil production and tissue accumulation at baseline or during infection with nematodes.

\section{Materials and methods}

\section{Mice}

Unless otherwise specified, IL-3 -/- mice were fourth backcross generation on the $\mathrm{C} 57 \mathrm{BL} / 6$ or BALB/c background. ${ }^{21,22} \mathrm{Kit}^{W} / \mathrm{Kit}^{W-v}$, IL-3 -/- mice were produced as previously described. ${ }^{22}$ Briefly, WBB6F1-Kit ${ }^{W /+}$ and $\mathrm{Kit}^{W-\mathrm{V} /+}$ mice were bred with
C57BL/6-IL-3 -/ - mice and the appropriate F2 and F3 offspring were identified and bred selectively to generate double-mutant mice; WBB6F1-Kit $+/+$ mice (Jackson Laboratory) were used as controls. All mouse experiments were conducted according to guidelines of the AAALAC-accredited BIDMC IACUC and NIH.

\section{Infection with Parasites}

S. venezuelensis (S.v.) or Nippostrongylus brasiliensis (N.b.) were maintained by serial passage in male Wistar rats or female BALB/C mice, respectively. Third-stage infective larvae (L3) of S.v. were obtained by fecal culture on filter paper, ${ }^{28}$ whereas L3 of N.b. were obtained by the charcoal-vermiculite fecal culture method. ${ }^{29}$ Mice were infected by subcutaneous (s.c.) inoculation with 400, 2000 or 10000 L3 of S.V., or were infected by s.c. inoculation with 600 L3 of N.b.

\section{Counting of Blood Leukocytes}

Total leukocytes were counted in samples of retroorbital blood using the UNOPETTE Test Kit (Becton Dickinson and Company, Franklin, NJ, USA). Smears were prepared of retro-orbital blood and were stained with May-Grünwald-Giemsa. A 300cell leukocyte differential count was performed and the number of eosinophils was calculated with reference to the total leukocyte count for that animal. Bone marrow cells were obtained by washing the cells from a single femur from each mouse and cytospin preparations of these cells were stained with May-Grünwald-Giemsa. The percentage of eosinophils in 500 bone marrow cells was determined for each sample. Bone marrow basophils were identified and quantified by flow cytometry. ${ }^{30}$

\section{Counting of Eosinophils and Mast Cells in Jejunum, Ileum, Spleen and Lung}

Mice were killed by $\mathrm{CO}_{2}$ inhalation before parasite infection ('baseline') or on various days after parasite infection and segments of jejunum and ileum were taken from an area $8-10 \mathrm{~cm}$ distal to the pylorus or from an area $4-6 \mathrm{~cm}$ proximal to the ileocecal junction, respectively. Segments of intestine, lung (taken near the main bronchus) and spleen were fixed in either Carnoy's fluid or in $10 \%$ neutral buffered formalin, $\mathrm{pH}$ 7.4. The fixed tissues were embedded in paraffin and $4 \mu \mathrm{m}$ tissue sections were deparaffinized and dehydrated. To demonstrate mast cells, the Carnoy's fixed sections were stained with $1 \%$ alcian blue, $\mathrm{pH} 1.0$ (spleen) or $0.5 \%$ alcian blue, $\mathrm{pH} 0.3$ (jejunum and ileum) at $37^{\circ} \mathrm{C}$ for $1 \mathrm{~h} .^{22}$ They were rinsed briefly in distilled water and counterstained with eosin-Y for $30 \mathrm{~s}$ and then dehydrated and mounted. To demonstrate 
eosinophils, the formalin-fixed sections were stained with hematoxylin-2 (Richard-Allan Scientific, Kalamazoo, MI, USA) for $5 \mathrm{~s}$ then rinsed briefly in distilled water and immersed in alkaline sodium chloride solution $(1.0 \%$ sodium hydroxide and saturated sodium chloride in $80 \%$ ethanol) for $20 \mathrm{~min}$ and stained in $0.5 \%$ Congo red solution (0.5\% Congo red in 50\% ethanol) for $20 \mathrm{~min}$ and dehydrated and mounted. ${ }^{31}$ Mast cells and eosinophils were counted by light microscopy by a single observer who was unaware of the identity (mouse genotype) of the individual sections. Mast cells and eosinophils in the jejunum and ileum were counted in at least 10 consecutive villus crypt units and the results were expressed as the mean $( \pm$ s.e.m.) cells per villus crypt unit. The spleen and lung mast cells or eosinophils were counted in 4-6 randomly selected sections and the results were expressed as the mean $\left( \pm\right.$ s.e.m.) cells per $\mathrm{mm}^{2}$.

\section{Statistics}

The data were analyzed for statistical significance (defined as $P<0.05$ ) using the two-tailed unpaired Student's $t$-test; unless otherwise specified, all data are expressed as mean \pm s.e.m.

\section{Results}

IL-3 - /- Mice Exhibit Normal Eosinophil Levels at Baseline and Unimpaired Eosinophil Responses to Infection with the Nematodes S.v. or N.b.

S.v. is a naturally occurring parasite of murine rodents that is rejected by a T-cell-dependent immune response which is associated with extensive mast cell hyperplasia and eosinophil infiltration in the affected intestinal mucosa. ${ }^{32-34}$ We reported that IL-3 -/- mice infected with 2000 S.V. L3, in comparison with the corresponding wildtype mice, exhibited significantly prolonged pro- duction of the parasites' eggs, as well as two marked abnormalities in their hematopoietic effector cell response to the infection: a virtual lack of parasiteinduced hyperplasia of bone marrow basophils and a significant reduction in parasite-induced mast cell hyperplasia in the intestines and spleen. ${ }^{22}$

When tissues derived from some of the mice used in the study of Lantz et $a 1^{22}$ were processed into Congo red-stained sections, we found that BALB/c IL-3 - / - and IL-3 + / + mice that were infected with 2000 S.v. L3 exhibited nearly identical levels of tissue eosinophilia in the jejunum, ( $\sim 2$-fold the corresponding levels in uninfected control mice), as well as similar levels of blood eosinophilia (Table 1). Similar results were obtained in a different experiment with C57BL/6 IL-3 -/ - and IL-3 + / + mice (Table 1).

To address the possibility that eosinophil responses in IL-3 $-/-$ mice might differ from those of IL-3 $+/+$ mice when the animals were infected with numbers of parasites that are different from that (ie, 2000 L3) used in our standard experiments, we infected groups of C57BL/6 IL-3 -/ - or IL-3 + / + mice with 400 or 10000 S.v. L3. No parasite egg production was observed in any of the mice infected with 400 S.v. L3, indicating that this number of S.v. failed to establish a detectable infection. However, as shown in Figure 1a, substantial parasite egg production occurred in IL-3 -/ - or IL-3 + / + mice that were infected with 10000 S.v. L3. In accord with our findings in mice infected with 2000 S.v. L3 ${ }^{22}$ the IL-3 - / - mice exhibited a significant delay in their ability to clear the infection, with parasite egg counts dropping to undetectable levels on day 15 of infection in the IL-3 -/- mice vs day 12 for the wild-type mice.

However, on days 7 and 8 of the infection with 10000 S.v. L3, parasite egg production in the IL-3 + / + mice was $\sim 3$-fold that in the IL-3 -/mice. This finding, that was not observed in IL-3 -/and IL-3 + / + mice which had been infected with 2000 S.v. L3, ${ }^{22}$ indicates that the effects of IL-3

Table 1 Tissue and blood eosinophil numbers in wild-type (IL-3 +/+) vs IL-3 -/ - mice, with or without infection with S.v.

\begin{tabular}{|c|c|c|c|c|}
\hline \multirow[t]{3}{*}{ Mice } & \multicolumn{4}{|c|}{ Eosinophils } \\
\hline & \multicolumn{2}{|c|}{ Jejunum (number/vcu) } & \multicolumn{2}{|c|}{ Blood (number/mm $\mathrm{mm}^{3}$ ) } \\
\hline & Control & 2000 S.v. & Control & 2000 S.v. \\
\hline \multicolumn{5}{|l|}{$B A L B / C$} \\
\hline IL-3 +/+ & $12 \pm 0.7(n=3)$ & $24 \pm 0.4^{\ddagger}(n=4)$ & $63 \pm 7(n=4)$ & $120 \pm 6^{\dagger}(n=4)$ \\
\hline IL-3 -/- & $12 \pm 1.0(n=3)$ & $25 \pm 0.6^{\ddagger}(n=4)$ & $57 \pm 3(n=8)$ & $103 \pm 7^{\ddagger}(n=4)$ \\
\hline \multicolumn{5}{|l|}{$C 57 B L / 6$} \\
\hline IL-3 +/+ & $8.5 \pm 0.5(n=3)$ & $16 \pm 0.5^{\ddagger}(n=8)$ & $50 \pm 5(n=8)$ & $192 \pm 28^{\dagger}(n=8)$ \\
\hline IL-3 -/- & $9.2 \pm 0.8(n=3)$ & $17 \pm 0.4^{\dagger}(n=3)$ & $52 \pm 11(n=3)$ & $124 \pm 25^{*}(n=3)$ \\
\hline
\end{tabular}

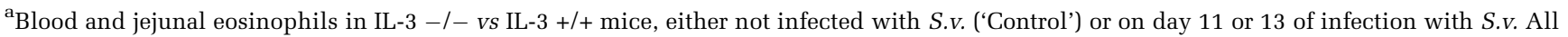
data are mean \pm s.e.m.

${ }^{*} P<0.05,{ }^{\dagger} P<0.001,{ }^{\ddagger} P<0.0001$ vs the corresponding baseline values. 

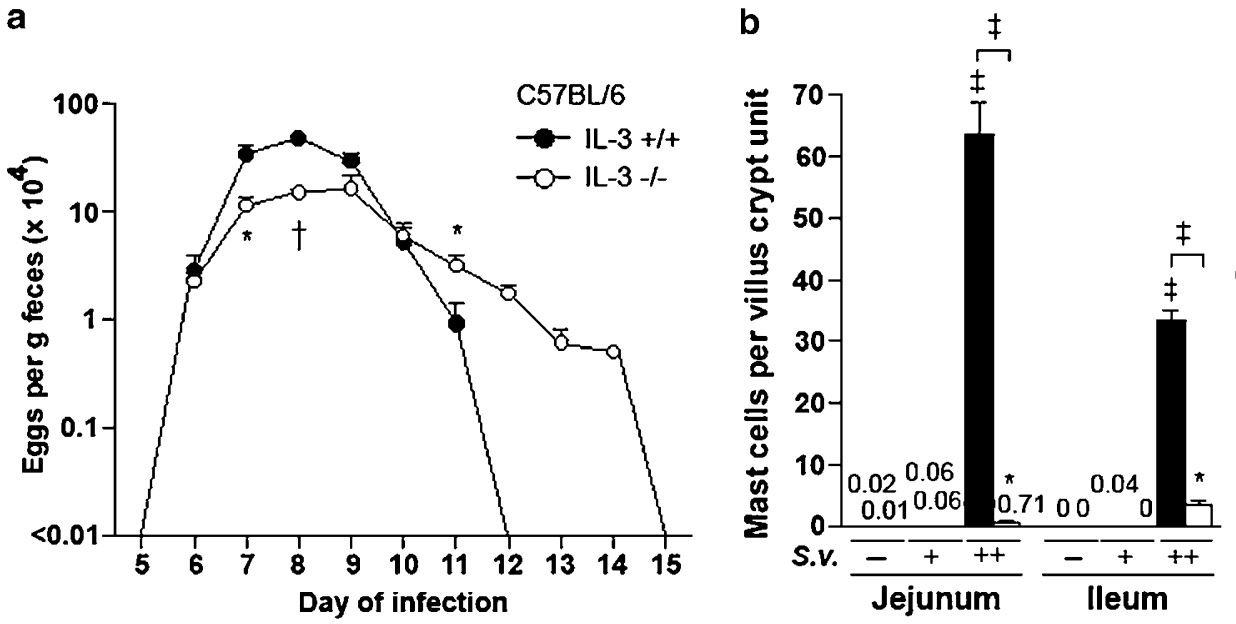

C57BL/6

C

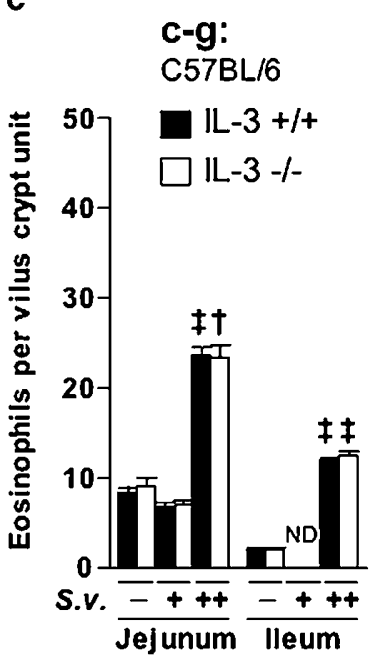

d

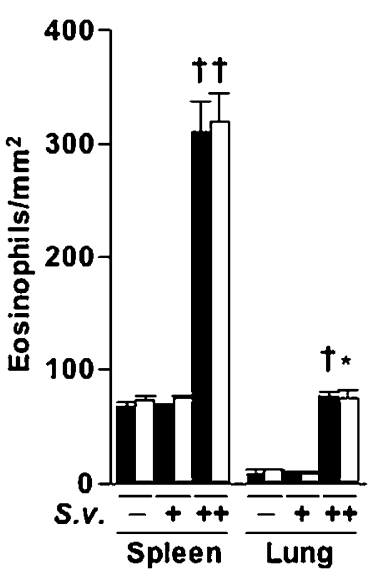

e

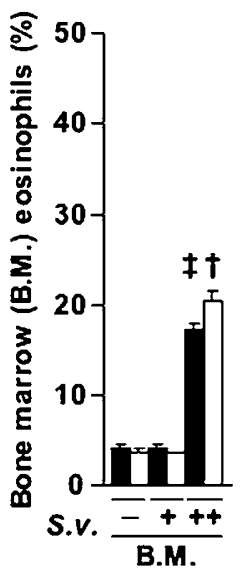

f

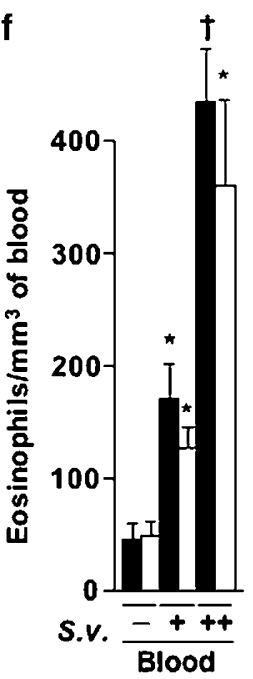

$\mathrm{IL}-3+/+$

IL-3 -

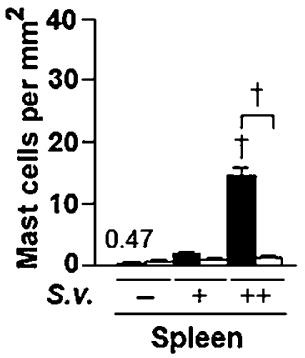

g

Figure 1 (a) Daily fecal egg production in female 8 to 10-week-old C57BL/6 IL-3 + / + vs IL-3 - / - mice after infection with 10000 L3 of $S$.v. All data are mean \pm s.e.m. ( $n=3-6$ per group). ${ }^{*} P<0.05,{ }^{\dagger} P<0.001$ vs corresponding values for IL-3 $+/+$ mice. All mice were killed on the day of clearance of infection. (b-g) Numbers of mast cells (b), eosinophils per villus crypt unit in jejunum and ileum (c), eosinophils per $\mathrm{mm}^{2}$ in sections of spleen and lung (d), \% of bone marrow (BM) eosinophils (e), and numbers of eosinophils (f), and WBC (white blood cells) (g) per $\mathrm{mm}^{3}$ of blood, at baseline (-) or after infection with $400(+)$ or $10000(++)$ L3 of S.v. in 8 to 10-week-old female C57BL/6 IL-3 + / + vs IL-3 - /- mice. All data are mean \pm s.e.m. ( $n=3-6$ per group), except that only mean values are given for very low values. ${ }^{*} P<0.05,{ }^{\dagger} P<0.001,{ }^{\ddagger} P<0.0001$ vs corresponding baseline values for mice of the same genotype or (as indicated by brackets) vs corresponding values for mice of the other genotype. Data for mice infected with $10000 \mathrm{~L} 3$ are from the same mice shown in (a). ND means not done.

during primary infection with S.v. may be quite complex: IL-3 can contribute to the rapid resolution of the infection, but, under certain circumstances, IL-3 may also enhance egg production at early stages of the infection.

In accord with results obtained in mice infected with 2000 S.v. L3, ${ }^{22}$ IL-3 $+/+$ mice infected with 10000 S.v. L3 exhibited a significant increase in numbers of bone marrow basophils by the time parasite egg expulsion was complete, whereas the identically challenged IL-3 $-/-$ mice did not $(2.4 \pm 0.2$ vs $0.86 \pm 0.05 \%$ basophils, $P<0.001)$. By contrast, infection with 400 S.v. L3 produced no significant changes in bone marrow basophil levels $(0.64 \pm 0.06$ or $0.65 \pm 0.02 \%$ in infected IL-3 $+/+$ or IL-3 -/ - mice, vs corresponding baseline values in uninfected IL-3 + / + or IL-3 - / - mice of $0.60 \pm 0.04$ or $0.55 \pm 0.08 \%$ ). Infection with 10000 S.v. L3 also induced a substantial mast cell hyperplasia in the jejunum and ileum of the IL- $3+/+$ mice, and resulted in greatly increased numbers of mast cells in the spleen, whereas the changes in mast cell numbers at these sites in the IL-3 -/ - mice were minimal (Figure 1b).

In contrast to the dramatic differences exhibited by IL-3 - / - and IL-3 + / + mice in changes in bone marrow basophil or tissue mast cell levels during S.v. infection, the eosinophil responses to infection in mice of either genotype (and the baseline eosinophil levels in uninfected mice) were statistically indistinguishable (Figure 1c-f). Blood eosinophil levels were slightly lower after S.v. infection in 
IL-3-/ - mice than in IL-3+/+ mice, but this difference was not statistically significant (Figure 1f); eosinophil levels in all of the other tested sites were virtually identical in the IL-3-/ - and IL-3 $+/+$ mice (Figure 1c-e). Note that mice infected with only 400 S.v. L3 also developed a significant blood eosinophilia (albeit to levels substantially lower than those in mice infected with $10000 \mathrm{L3}$ ), and the levels in the IL-3-/- mice were slightly, but not significantly lower than those in the IL- $3+/+$ mice (Figure 1f). Thus, of the three hematopoietic lineages examined (eosinophils, basophils, and mast cells), the eosinophil was the most sensitive indicator of the presence of parasites.

We also examined eosinophil responses in mice infected with the nematode N.b., which, like S.v., induces a Th2-type immune response that is associated with blood and tissue eosinophilia. ${ }^{32,35}$ However, even though mice infected with N.b. develop an intestinal mast cell hyperplasia, several lines of evidence indicate that mast cells have little or no role in the resolution of N.b. infection. ${ }^{34-36} \mathrm{We}$ found that BALB/C IL-3-/- or IL- $3+/+$ mice infected with 600 N.b. L3 exhibited a virtually identical pattern of parasite egg production, with complete clearance of the infection in mice of either genotype by day 11 (data not shown). However, as in mice infected with S.v., N.b.-infected IL-3+/+ mice developed increased numbers of bone marrow basophils ( $\sim 100 \%$ increase) and increased numbers of mast cells in the jejunum, ileum and spleen, whereas the N.b.-infected IL-3-/ - mice failed to develop increased numbers of bone marrow basophils and exhibited greatly impaired expansion of intestinal and splenic mast cell populations (data not shown). By contrast, both IL-3-/ - and IL-3 + / + mice developed substantial, and virtually identical, increases in levels of bone marrow, blood, jejunal, ileal and splenic eosinophils in response to N.b. infection (Figure 2).

These results strongly support the conclusion that, despite its well-documented ability to promote eosinophil development in vitro, IL-3 can be dispensable for eosinophil production and tissue accumulation in mice in vivo. In fact, we have shown using a mouse model of ragweed-induced allergic inflammation known to be associated with a selective tissue accumulation of eosinophils that, under some circumstances, IL-3 can actually have a suppressive effect on eosinophil development and/ or migration. ${ }^{19}$ While the potential role of IL-3 in regulating eosinophil production or tissue accumulation of eosinophils in the context of S.V. infection had not previously been investigated, Madden et $a l^{32}$ examined the effects of the administration of polyclonal antibodies to mouse IL-3 on the blood eosinophilia, and enhanced levels of tissue mast cells, which developed in mice that were infected with N.b. They found that treatment with anti-IL-3 antibodies reduced N.b.-induced increases in tissue mast cells by $\sim 40 \%$, but had no detectable effect on blood eosinophilia on days 11 or 13 of the infection. ${ }^{32}$

The results of this study, and those reported by Lantz et $a{ }^{22}{ }^{22}$ clearly show that the requirements for IL-3 for expansion of basophil, intestinal mast cell and eosinophil populations during Th2-type responses to intestinal helminths in mice are distinct; IL-3 is required for the basophil response and importantly contributes to the mast cell response, but makes little or no detectable contribution to the eosinophil response. This conclusion is supported by our findings derived from mice which had been subjected to infection with a wide range of S.v. third
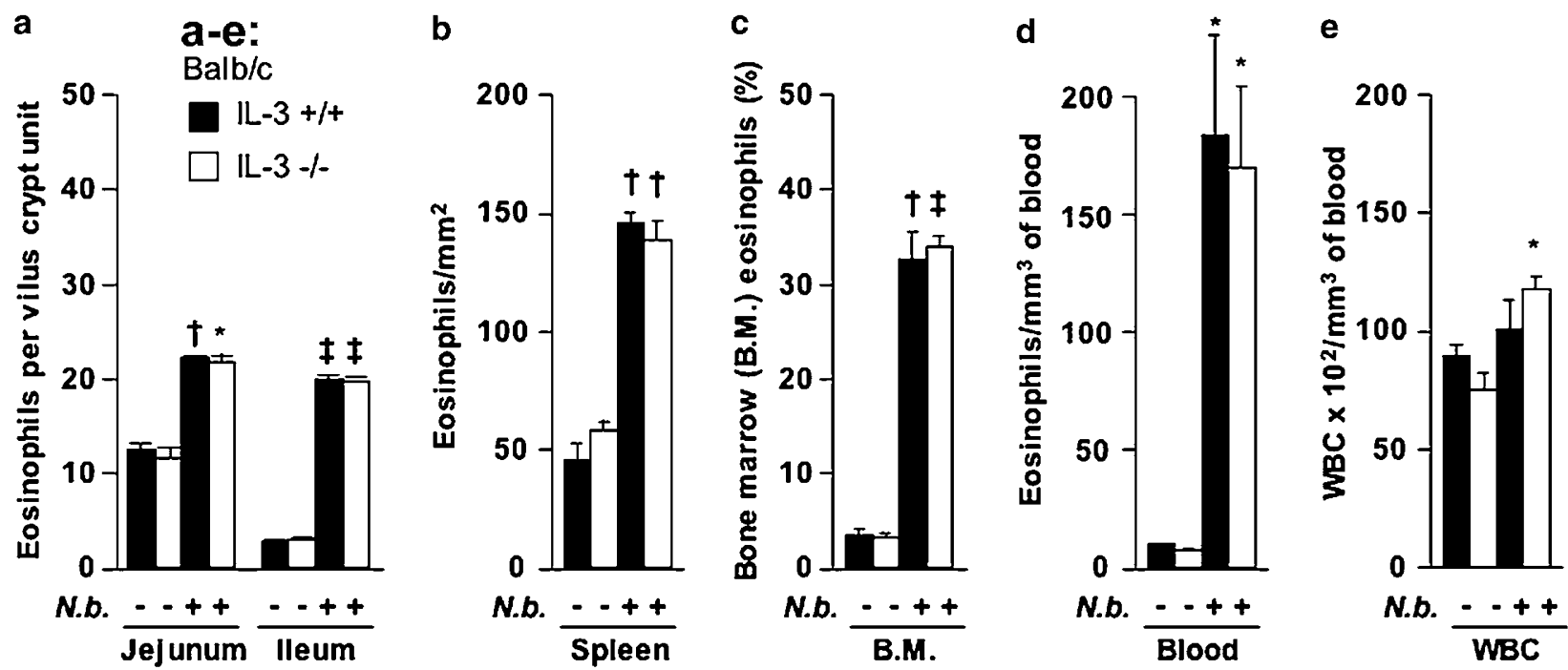

Figure 2 Numbers of eosinophils per villus crypt unit in jejunum and ileum (a), eosinophils per $\mathrm{mm}^{2}$ in sections of spleen (b), \% of bone marrow (BM) eosinophils (c), and numbers of eosinophils (d) and WBC (white blood cells) (e) per mm ${ }^{3}$ of blood, at baseline (-) or at day 11 after infection with 600 L3 of N.b. in 3 to 4-month-old male or female BALB/c IL-3 + / + vs IL-3 -/ - mice. All data are mean \pm s.e.m. ( $n=3-5$ per group). ${ }^{*} P<0.05$, ${ }^{\dagger} P<0.001,{ }^{\ddagger} P<0.0001$ vs the corresponding baseline values for mice of the same genotype. 
stage larvae: 400,2000 or 10000 , as well as by our results and those of Madden et $a l^{32}$ in N.b.-infected mice.

Infection with S.v., but not N.b., Induces Substantially Less Tissue Accumulation of Eosinophils in

$\mathrm{Kit}^{W} / \mathrm{Kit}^{W-v}, \mathrm{IL}-3-/-$ Mice Than in

$\mathrm{Kit}^{W} / \mathrm{Kit}^{\mathrm{W-v}}, \mathrm{IL-3}+\mathrm{I}+\mathrm{Mice}$

Our findings in IL-3- $/-$ and $+/+$ mice do not exclude the possibility that IL-3 might contribute to eosinophil responses in settings different than those we tested. For example, we reported that mast cell development and parasite immunity are impaired even more severely in S.v.-infected IL-3-/- mice that have markedly reducing c-Kit/SCF signaling (ie $\mathrm{Kit}^{W} / \mathrm{Kit}^{\mathrm{W-v}}$, IL-3 $-/-$ mice) than in similarly

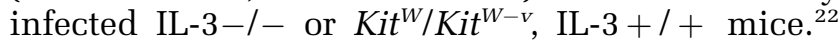
We found that baseline numbers of blood, bone marrow and tissue eosinophils were essentially identical in $\mathrm{Kit}^{W} / \mathrm{Kit}^{W-v}$, IL-3-/- and $\mathrm{Kit}^{W} / \mathrm{Kit}^{\mathrm{W-v}}$, IL-3 $+/+$ mice (Figure 3). By contrast, S.v. infection induced a significant increase in tissue eosinophil levels in $\mathrm{Kit}^{W} / \mathrm{Kit}^{W-\mathrm{v}}$, IL-3 + I+ mice but, with the exception of the bone marrow, not in the $\mathrm{Kit}^{W} / \mathrm{Kit}^{\mathrm{W-v}}$, IL-3-/ - mice (Figure 3). Although blood eosinophil levels were comparable in uninfected and S.v.infected $\mathrm{Kit}^{W} / \mathrm{Kit}^{\mathrm{W-v}}$, IL-3 + / + mice, blood eosinophil levels were significantly lower in S.v.-infected

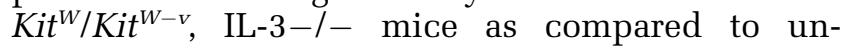

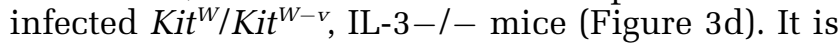
important to note that all mice were killed when $\mathrm{Kit}^{W} / \mathrm{Kit}^{W-v}$, IL-3 + / + mice cleared their infection (days 18 or 19), a point in the infection in which $\mathrm{Kit}^{W} / \mathrm{Kit}^{W-v}$, IL-3-/- mice still retained most of their parasites. ${ }^{22}$ Thus, Kit ${ }^{W} / \mathrm{Kit}^{W-v}$, IL-3-/- mice exhibited a significantly impaired tissue eosinophil response despite the persistent stimulus of a heavy parasite burden.
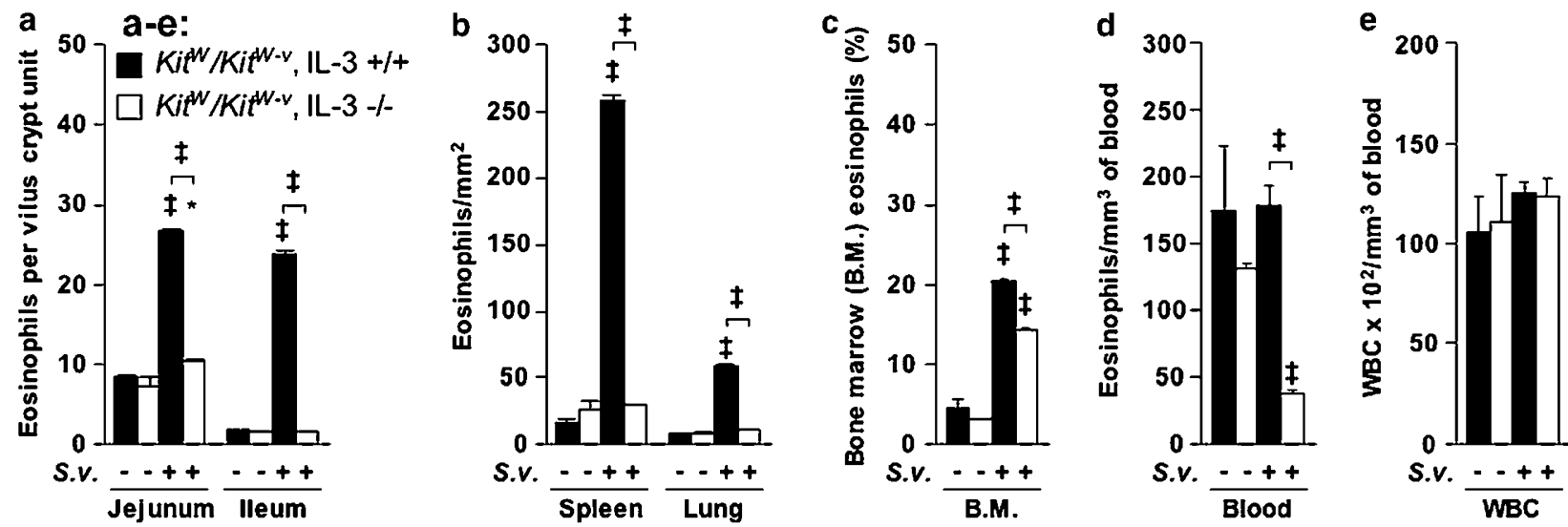

Figure 3 Numbers of eosinophils per villus crypt unit in jejunum and ileum (a), eosinophils per $\mathrm{mm}^{2}$ in sections of spleen and lung (b), $\%$ of bone marrow (BM) eosinophils (c), and numbers of eosinophils (d) and WBC (white blood cells) (e) per mm ${ }^{3}$ of blood, at baseline ( - ) or at days 18 or 19 after infection with $2000 \mathrm{~L} 3$ of S.v. in 3 to 4 -month-old male or female Kit ${ }^{W} / K_{i t}^{W-v}$, IL-3 $+/+v s \mathrm{Kit}^{W} / \mathrm{Kit}^{W-v}$, IL-3 -/mice. The data for S.v.-infected mice are from the same groups of mice depicted in Figure 3a in Lantz et al. ${ }^{22}$ All data are mean \pm s.e.m. ( $n=3-9$ per group). ${ }^{*} P<0.05,{ }^{\ddagger} P<0.0001$ vs the corresponding baseline values for mice of the same genotype or (as indicated by brackets) vs corresponding values for mice of the other genotype. 
a

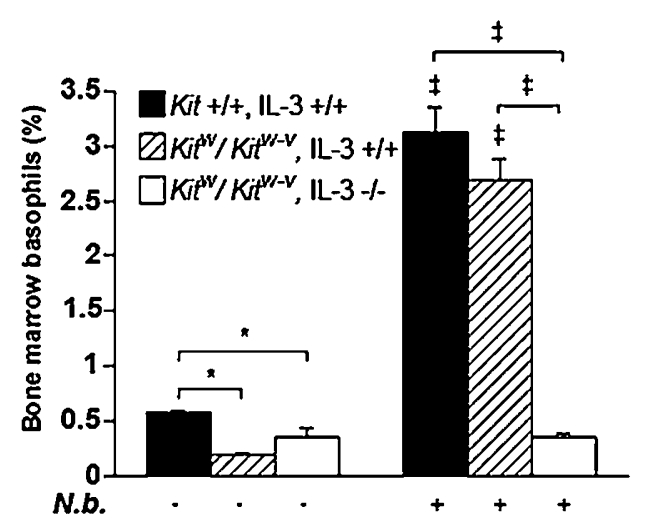

b

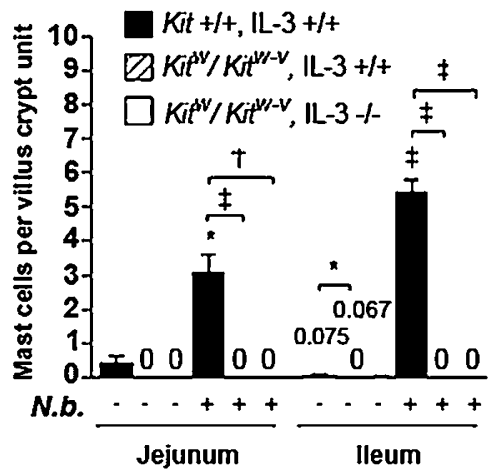

C

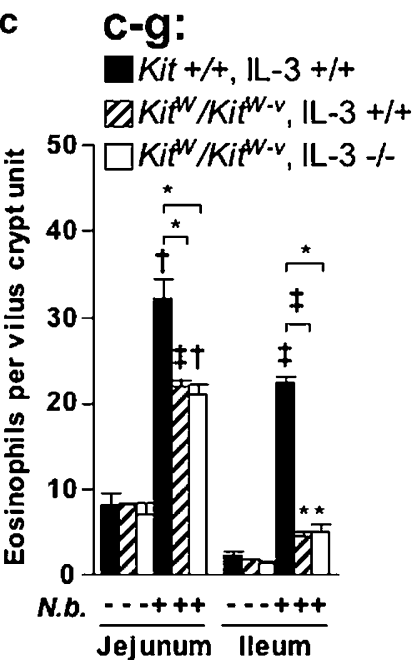

d

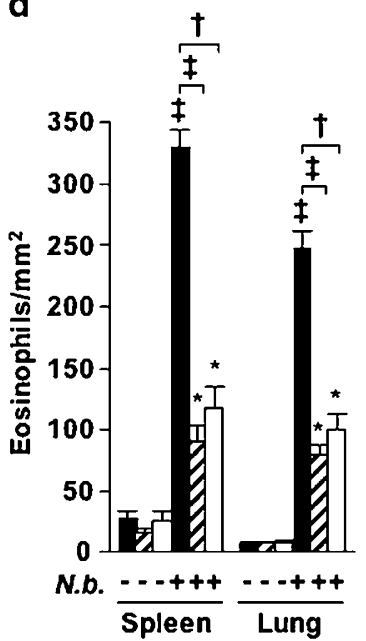

e

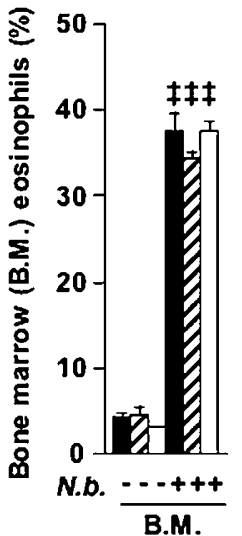

f

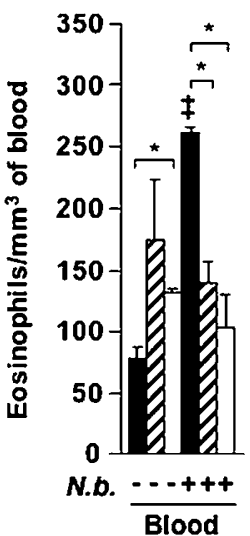

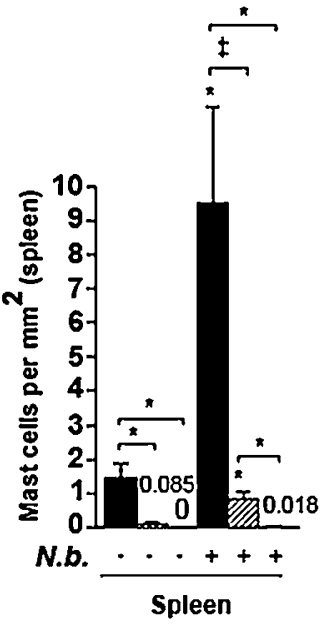

g

Figure 4 (a-g) Percentage of bone marrow basophils (a), numbers of mast cells (b), and numbers of eosinophils per villus crypt unit in jejunum and ileum (c), eosinophils per $\mathrm{mm}^{2}$ in sections of spleen and lung (d), \% of bone marrow (BM) eosinophils (e), and numbers of eosinophils (f) and WBC (white blood cells) (g) per $\mathrm{mm}^{3}$ of blood, at baseline (-) or at days 11-14 after infection with 600 L3 of N.b. in 3

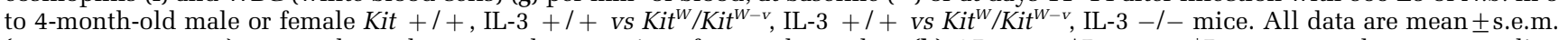
( $n=3-10$ per group), except that only mean values are given for very low values (b). ${ }^{\star} P<0.05,{ }^{\dagger} P<0.001,{ }^{\ddagger} P<0.0001$ vs the corresponding baseline values for mice of the same genotype or (as indicated by brackets) vs corresponding values for mice of another genotype. Baseline data for uninfected mice are from the same groups of mice depicted in Figure 3d in Lantz et al ${ }^{22}$ (a and b) or from Figure 3(c-g).

nematodes remains to be determined. Wild-type mice were not infected with S.v. during the experiment shown in Figure 3, however, the eosinophil responses the S.V.-infected $\mathrm{Kit}^{W} / \mathrm{Kit}^{W-v}$, IL-3+ /+ mice depicted in Figure 3a-d were similar to those observed in the S.v.-infected wild-type (IL-3+/+) mice shown in Table 1 and Figure 1c-f. Taken together, these results indicate that the eosinophil responses to N.b. infection in mice may be more dependent on C-Kit than are those observed during infection with S.v.

Table 2 summarizes some of our key findings regarding the roles of IL-3 and c-Kit signaling in influencing the numbers of blood and tissue eosinophils in mice subjected to the two different models of parasite infection analyzed in this study.

\section{Discussion}

IL-3 appeared to make no detectable contribution to eosinophil numbers in normal mice, in that we found no significant differences in the numbers of eosinophils in the blood, bone marrow or various tissues of IL-3-/ - vs IL-3 $+/+$ mice, either at baseline or after the induction of bone marrow, blood or tissue eosinophilia in response to infection with S.v. or N.b. However, in $\mathrm{Kit}^{W} / \mathrm{Kit}^{W-v}$ mice, which have markedly impaired SCF/c-Kit signaling, our results indicate that IL-3 contributed significantly to the increased numbers of eosinophils that were observed in multiple tissues during S.v. infection, but not during infection with N.b.

A number of mechanisms may contribute to the

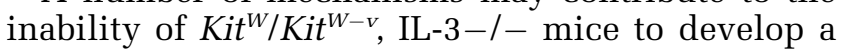


Table 2 Key findings regarding the influence of IL-3 and/or c-Kit signaling on levels of blood or tissue eosinophils in mice infected with S.v. or N.b.

Setting Key findings

1. IL-3 deficiency in otherwise normal mice infected with S.v. or N.b.

2. IL-3 deficiency in $\mathrm{Kit}^{W} / \mathrm{Kit}^{\mathrm{W-V}}$ mice infected with S.v.

3. IL-3 deficiency in $\mathrm{Kit}^{W} / \mathrm{Kit}^{\mathrm{W-V}}$ mice infected with N.b.
At the times after infection analyzed, a lack of IL-3 had no significant effect on the substantially increased numbers of blood eosinophils, \% of bone marrow eosinophils, or numbers of tissue eosinophils (in jejunum, ileum or spleen) in C57BL/6 mice infected with $10000 \mathrm{~L} 3$ of S.v. (Figure 1) or in BALB/C mice infected with 600 L3 of N.b. (Figure 2); IL-3 also had no significant effect on the increased numbers of blood eosinophils or numbers of eosinophils in the jejunum in C57BL/6 or BALB/c mice infected with 2000 L3 of S.v. (Table 1).

These findings indicate that, under the conditions examined, the presence or absence of IL-3 in otherwise normal C57BL/6 or BALB/c mice had little or no effect on the increased numbers of bone marrow, blood or tissue eosinophils which were observed as a result of infection with either of the two species of nematodes analyzed.

At the times after infection analyzed, a lack of IL-3 markedly and significantly reduced the increases in the numbers of eosinophils in the jejunum, ileum, spleen and lung that were observed as a result of infection of $\mathrm{Kit}^{W} / \mathrm{Kit}^{W-v}$ mice (that are markedly defective in c-Kit signaling) with $2000 \mathrm{~L} 3$ of S.v. (Figure 3); a lack of IL-3 in $\mathrm{Kit}^{W} / \mathrm{Kit}^{W-V}$ mice also resulted in significantly fewer blood eosinophils and a significantly smaller increase in the \% of bone marrow eosinophils that were observed during such infection (Figure 3).

These findings indicate that, in mice with markedly diminished c-Kit signaling, IL-3 can contribute significantly to the increases in bone marrow and, particularly, tissue, eosinophils associated with infection with S.v., at least under the conditions tested.

At days 11-14 after infection with 600 L3 of N.b., the presence or absence of IL-3 had little or no additional effect on the significant reductions in the elevated levels of tissue (jejunum, ileum, spleen or lung) or blood eosinophils which occurred in $\mathrm{Kit}^{W} / \mathrm{Kit}^{W-V}$ mice (with markedly defective c-Kit signaling) as compared to the corresponding values in wild-type mice; however, the \% of bone marrow eosinophils was elevated to the same extent in N.b.-infected wild-type, $\mathrm{Kit}^{W} / \mathrm{Kit}^{W-v}$ or $\mathrm{Kit}^{W} / \mathrm{Kit}^{W-v}$, IL-3 - / - mice (Figure 4).

These findings indicate that mice with markedly diminished c-Kit signaling exhibit significant reductions in the numbers of blood and tissue, but not bone marrow, eosinophils associated with infection with $N . b$. , but that the presence or absence of IL-3 has little or no effect on the values for these eosinophil populations in $\mathrm{Kit}^{W} / \mathrm{Kit}^{W-V}$ mice, at least under the conditions tested. robust eosinophil response to S.v. IL-3 can expand numbers of eosinophils generated from hematopoietic stem cells in vitro ${ }^{2}$, an effect which can be enhanced by SCF. ${ }^{23,24}$ Therefore, both IL-3 and SCF may be required for increased commitment of progenitor cells to the eosinophilic lineage during a primary infection with S.v. Alternatively, or in addition, IL-3 and SCF may be required to promote eosinophil accumulation during S.v. infection by influencing eosinophil migration. Indeed, Yuan et $a l^{25}$ have provided evidence that SCF is an agonist for eosinophil adhesion to endothelial cells. Other studies suggest that eosinophil migration is in part regulated by the ability of resident mast cells to secrete chemoattractants for eosinophils, such as eotaxin and leukotrienes. ${ }^{37-40}$ Modest numbers of mast cells do develop in the intestinal mucosal tissue or spleen of $\mathrm{Kit}^{W} / \mathrm{Kit}^{\mathrm{W-v}}$, IL-3+/+ mice during infection with S.v., ${ }^{22,33}$ however, mast cell are virtually absent from the corresponding tissues of S.V.-infected $\mathrm{Kit}^{W} / \mathrm{Kit}^{\mathrm{W-v}}$, IL-3-/- mice. ${ }^{22}$ Thus, the presence of low levels of eosinophils in S.V.-

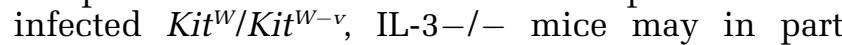
reflect the virtual absence of mast cells in these animals.

Our findings show that IL-3 can cooperate with c-Kit/SCF to increase numbers of eosinophils in response to S.v. infection. This is clearly shown in Figure 3, which demonstrates that the ability of S.v. infection to enhance eosinophil numbers in the tissues is almost undetectable in $\mathrm{Kit}^{W} / \mathrm{Kit}^{\mathrm{W}-\mathrm{v}}$, IL-3-/- mice. Although the precise mechanism(s) by which IL-3 and c-Kit/SCF regulate the eosinophil responses during S.v. infection remain(s) to be determined, one important implication of our findings is that the impaired immunity to S.V. expressed by $\mathrm{Kit}^{W} / \mathrm{Kit}^{W-v}$, IL-3-/- mice ${ }^{22}$ may in part reflect the combined contributions of mast cells, basophils and eosinophils.

We also noted that, on days 7 and 8 of the infection with 10000 S.v. L3, parasite egg production in IL- $3+/+$ mice was $\sim 3$-fold that in the IL-3-/ - mice. Thus, in addition to contributing to the rapid resolution of the infection, under certain circumstances, IL-3 may also have effects that can enhance egg production at early stages of the infection. While the explanation for this observation remains to be determined, two studies have shown that egg production during nematode infections can be significantly higher in rats which have normal numbers of mast cells than in rats which are mast cell-deficient either because of loss-of-function mutations in c-kit ${ }^{41}$ or because of mast cell depletion as a result of the administration of an anti-SCF antibody. ${ }^{42}$ In the context of these findings in the rat, and in light of evidence that IL-3 can both promote increased numbers of mast cells and basophils ${ }^{22}$ and also can enhance the function of these cells, ${ }^{43,44}$ as well as have effects on eosinophils, we can speculate about at least one mechanism by which 
IL-3 might promote increased nematode fecundity. Perhaps, in certain stages of such infections, the net effect of IL-3-dependent enhancement of mast cell, basophil and/or eosinophil numbers and/or function is to promote parasite fecundity. This might occur, for example, if IL-3 promoted the increased local release by mast cells, basophils and/or eosinophils of mediators that can enhance intestinal vascular or epithelial cell permeability, thereby favoring parasite nutrition, health and egg production.

\section{Acknowledgements}

This work was supported by N.I.H. Grants AI-23990 and CA-72074 (SJG), and AI-49932 (CSL). We thank Martin Metz, MD and Caroline Tudor for assistance in producing the figures.

\section{References}

1 Ihle JN. Interleukin-3 and hematopoiesis. Chem Immunol 1992;51:65-106.

2 Sanderson CJ, Warren DJ, Strath M. Identification of a lymphokine that stimulates eosinophil differentiation in vitro. Its relationship to interleukin 3, and functional properties of eosinophils produced in cultures. J Exp Med 1985;162:60-74.

3 Rothenberg ME, Owen Jr WF, Silberstein DS, et al. Human eosinophils have prolonged survival, enhanced functional properties, and become hypodense when exposed to human interleukin 3. J Clin Invest 1988;81:1986-1992.

4 Warren DJ, Moore MA. Synergism among interleukin 1, interleukin 3, and interleukin 5 in the production of eosinophils from primitive hemopoietic stem cells. J Immunol 1988;140:94-99.

5 Wardlaw AJ, Moqbel R, Kay AB. Eosinophils: biology and role in disease. Adv Immunol 1995;60:151-266.

6 Rothenberg ME. Eosinophilia. N Engl J Med 1998; 338:1592-1600.

7 Robb L, Drinkwater CC, Metcalf D, et al. Hematopoietic and lung abnormalities in mice with a null mutation of the common beta subunit of the receptors for granulocyte-macrophage colony-stimulating factor and interleukins 3 and 5. Proc Natl Acad Sci USA 1995;92: 9565-9569.

8 Nishinakamura R, Nakayama N, Hirabayashi Y, et al. Mice deficient for the IL-3/GM-CSF/IL-5 beta c receptor exhibit lung pathology and impaired immune response, while beta IL3 receptor-deficient mice are normal. Immunity 1995;2:211-222.

9 Nicola NA, Robb L, Metcalf D, et al. Functional inactivation in mice of the gene for the interleukin-3 (IL-3)-specific receptor beta-chain: implications for IL3 function and the mechanism of receptor transmodulation in hematopoietic cells. Blood 1996;87:26652674.

10 Nishinakamura R, Miyajima A, Mee PJ, et al. Hematopoiesis in mice lacking the entire granulocytemacrophage colony-stimulating factor/interleukin-3/ interleukin-5 functions. Blood 1996;88:2458-2464.
11 Hayashida K, Kitamura T, Gorman DM, et al. Molecular cloning of a second subunit of the receptor for human granulocyte-macrophage colony-stimulating factor (GM-CSF): reconstitution of a high-affinity GM-CSF receptor. Proc Natl Acad Sci USA 1990;87: 9655-9659.

12 Kitamura T, Sato N, Arai K, et al. Expression cloning of the human IL-3 receptor cDNA reveals a shared beta subunit for the human IL-3 and GM-CSF receptors. Cell 1991;66:1165-1174.

13 Gorman DM, Itoh N, Kitamura $\mathrm{T}$, et al. Cloning and expression of a gene encoding an interleukin 3 receptor-like protein: identification of another member of the cytokine receptor gene family. Proc Natl Acad Sci USA 1990;87:5459-5463.

14 Itoh N, Yonehara S, Schreurs J, et al. Cloning of an interleukin-3 receptor gene: a member of a distinct receptor gene family. Science 1990;247:324-327.

15 Hara T, Miyajima A. Two distinct functional high affinity receptors for mouse interleukin-3 (IL-3). EMBO J 1992;11:1875-1884.

16 Kopf M, Brombacher F, Hodgkin PD, et al. IL-5deficient mice have a developmental defect in CD5+ B-1 cells and lack eosinophilia but have normal antibody and cytotoxic $\mathrm{T}$ cell responses. Immunity 1996;4:15-24.

17 Yoshida T, Ikuta K, Sugaya H, et al. Defective B-1 cell development and impaired immunity against Angiostrongylus cantonensis in IL-5R alpha-deficient mice. Immunity 1996;4:483-494.

18 Takamoto M, Sugane K. Synergism of IL-3, IL-5, and GM-CSF on eosinophil differentiation and its application for an assay of murine IL-5 as an eosinophil differentiation factor. Immunol Lett 1995;45:43-46.

19 Neel NF, Creasy BM, Rankin JN, et al. Absence of interleukin-3 does not affect the severity of local and systemic anaphylaxis but does enhance eosinophil infiltration in a mouse model of allergic peritonitis. Immunol Lett 2004;95:37-44.

20 Gillessen S, Mach N, Small C, et al. Overlapping roles for granulocyte-macrophage colony-stimulating factor and interleukin-3 in eosinophil homeostasis and contact hypersensitivity. Blood 2001;97:922-928.

21 Mach N, Lantz CS, Galli SJ, et al. Involvement of interleukin-3 in delayed-type hypersensitivity. Blood 1998;91:778-783.

22 Lantz CS, Boesiger J, Song CH, et al. Role for interleukin-3 in mast-cell and basophil development and in immunity to parasites. Nature 1998;392: 90-93.

23 Kobayashi H. Effect of c-kit ligand (stem cell factor) in combination with interleukin-5, granulocyte-macrophage colony-stimulating factor, and interleukin-3, on eosinophil lineage. Int J Hematol 1993;58:21-26.

24 Metcalf D, Mifsud S, Di Rago L. Stem cell factor can stimulate the formation of eosinophils by two types of murine eosinophil progenitor cells. Stem Cells 2002; 20:460-469.

25 Yuan Q, Austen KF, Friend DS, et al. Human peripheral blood eosinophils express a functional ckit receptor for stem cell factor that stimulates very late antigen 4 (VLA-4)-mediated cell adhesion to fibronectin and vascular cell adhesion molecule 1 (VCAM-1). J Exp Med 1997;186:313-323.

26 Kitamura Y, Go S, Hatanaka K. Decrease of mast cells in $W / W^{v}$ mice and their increase by bone marrow transplantation. Blood 1978;52:447-452. 
27 Tsai M, Lantz CS, Galli SJ. Regulation of mast cell and basophil development by stem cell factor and interleukin-3. In: Marone G, Lichtenstein LM, Galli SJ (eds). Mast Cells and Basophils. Academic Press: London, 2000, pp 3-20.

28 Sato Y, Toma H. Strongyloides venezuelensis infections in mice. Int J Parasitol 1990;20:57-62.

29 Huff TF, Lantz CS. Culture of murine mast cells. In: Lefkovits I (ed). The Immunology Methods Manual, Vol. 3. Academic Press: London, 1997, pp 1393-1407.

30 Lantz CS, Yamaguchi M, Oettgen HC, et al. IgE regulates mouse basophil Fc epsilon RI expression in vivo. J Immunol 1997;158:2517-2521.

31 Grouls V, Helpap B. Selective staining of eosinophils and their immature precursors in tissue sections and autoradiographs with Congo red. Stain Technol 1981; 56:323-325.

32 Madden KB, Urban Jr JF, Ziltener HJ, et al. Antibodies to IL-3 and IL-4 suppress helminth-induced intestinal mastocytosis. J Immunol 1991;147:1387-1391.

33 Khan AI, Horii Y, Tiuria R, et al. Mucosal mast cells and the expulsive mechanisms of mice against Strongyloides venezuelensis. Int J Parasitol 1993;23: 551-555.

34 Nawa Y, Ishikawa N, Tsuchiya K, et al. Selective effector mechanisms for the expulsion of intestinal helminths. Parasite Immunol 1994;16:333-338.

35 Gause WC, Urban Jr JF, Stadecker MJ. The immune response to parasitic helminths: insights from murine models. Trends Immunol 2003;24:269-277.

36 Finkelman FD, Shea-Donohue T, Goldhill J, et al. Cytokine regulation of host defense against parasitic gastrointestinal nematodes: lessons from studies with rodent models. Annu Rev Immunol 1997;15:505-533.

37 Harris RR, Komater VA, Marett RA, et al. Effect of mast cell deficiency and leukotriene inhibition on the influx of eosinophils induced by eotaxin. J Leukoc Biol 1997;62:688-691.

38 Das AM, Flower RJ, Perretti M. Resident mast cells are important for eotaxin-induced eosinophil accumulation in vivo. J Leukoc Biol 1998;64:156-162.

39 Hogaboam C, Kunkel SL, Strieter RM, et al. Novel role of transmembrane SCF for mast cell activation and eotaxin production in mast cell-fibroblast interactions. J Immunol 1998;160:6166-6171.

40 Klein A, Talvani A, Silva PM, et al. Stem cell factorinduced leukotriene B4 production cooperates with eotaxin to mediate the recruitment of eosinophils during allergic pleurisy in mice. J Immunol 2001; 167:524-531.

41 Arizono N, Kasugai T, Yamada M, et al. Infection of Nippostrongylus brasiliensis induces development of mucosal-type but not connective tissue-type mast cells in genetically mast cell-deficient $W_{s} / W s$ rats. Blood 1993;81:2572-2578.

42 Newlands GF, Miller HR, MacKellar A, et al. Stem cell factor contributes to intestinal mucosal mast cell hyperplasia in rats infected with Nippostrongylus brasiliensis or Trichinella spiralis, but anti-stem cell factor treatment decreases parasite egg production during $N$ brasiliensis infection. Blood 1995;86: 1968-1976.

43 Lantz CS, Galli SJ. The role of mast cells, basophils and interleukin-3 (IL-3) in immune responses to parasites: Studies with mast cell- and IL-3-deficient mice. In: Marone G, Lichtenstein LM, Galli SJ (eds). Mast Cells and Basophils. Academic Press: London, 2000, pp 439-452.

44 Vilarino N, Miura K, MacGlashan Jr DW. Acute IL-3 priming up-regulates the stimulus-induced Raf-1-MekErk cascade independently of IL-3-induced activation of Erk. J Immunol 2005;175:3006-3014. 